\title{
Development of a pore gas pressure transducer used in unsaturated soils at high water content
}

\author{
Rui Chen ${ }^{1}$, Zhongkui Chen ${ }^{1, *}$, Charles Wang Wai $\mathrm{Ng}^{2}$ and Jian $\mathrm{Liu}^{3}$ \\ ${ }^{1}$ Department of Civil and Environmental Engineering, Harbin Institute of Technology, Shenzhen, 518055 Shenzhen, China \\ ${ }^{2}$ Department of Civil and Environmental Engineering, The Hong Kong University of Science and Technology, Kowloon, HKSAR \\ ${ }^{3}$ Geotechnical Research Institute, Hohai University, 1 Xikang Road, 210098 Nanjing, China
}

\begin{abstract}
Pore gas pressure in soil is an important parameter in many geoscience applications such as evaluating the effects of trapped pore gas pressure on water infiltration through soil mass, optimizing the design of gas extraction wells in landfills and assessing the performance of landfill covers in reducing landfill gas emission. In addition, it has been observed that pore gas pressure affects slope stability in unsaturated soils. However, the pore gas pressure build-up induced by water infiltration is generally ignored in most slope stability analysis by assuming gas pressure to be zero. Therefore, pore gas pressure measurement in soils is crucial to better understand the unsaturated soil behaviour. However, most of current measuring techniques of pore gas pressure are affected by water interruption during the measurement in unsaturated soils, especially at high water content. In this study, a novel gas pressure transducer was developed to measure the pore gas pressure in unsaturated soil within a wide range of water content. The newly developed pore gas pressure transducer mainly consists of an electrical pressure sensor package and an integrated membrane filter which can prevent water leaching through the membrane but allow gas to pass it freely. The performance of the gas pressure transducer was evaluated by a series of permeation tests. The results show that the developed gas pressure transducer has a good repeatability to monitor gas pressure and has a relatively fast response to the gas pressure change in compacted soils. This transducer is able to measure pore gas pressure range of $0 \sim 50$ $\mathrm{kPa}$ of soils within a relatively high range of soil water content.
\end{abstract}

\section{Introduction}

Pore gas pressure in soil is an important parameter in many geoscience applications such as evaluating the effects of pore trapped gas pressure on water infiltration through soil mass [1-2], optimizing the design of gas extraction wells in landfills, assessing the performance of a landfill cover in reducing landfill gas emission [3] and providing high quality data to verify numerical models. In addition, it has been observed that pore gas pressure affects slope stability analysis in unsaturated soils [4]. However, the excess pore gas pressure induced by water infiltration is generally ignored in most slope stability analysis by assuming gas pressure to be maintained at zero. This is mainly due to the assumption that pore gas in soil mass is directly open to the atmosphere. However, in reality, the gas inside the unsaturated soil is not fully connected with the atmosphere, but partly trapped inside the soil depending on the soil degree of saturation [5]. Therefore, gas pressure measurement in soils is important to better understand the unsaturated soil behaviour.

To measure pore gas pressure in soils, there are two common measuring techniques: U-tube and pressure transducer-based method. U-tube is an instrument that uses a column of liquid with a U-shape to measure gas pressure. It is mainly based on the physical pressure balance through the liquid (i.e., water) at two ends of the tube. Normally, one end of the tube is exposed to the atmosphere and the other is connected with the medium containing gas pressure. It has been used to measure landfill gas pressure in laboratory column tests [6]. However, this type of technique cannot prevent water in soil from permeating into the measuring tube, especially in a high degree of saturation of soil, resulting in unreliable gas pressure measurement. In addition, this type of measuring technique is hard to apply to high gas pressure range (for instance, the extreme landfill gas pressure may reach up to $50 \mathrm{kPa}$ ) due to the limit of available tube length.

Vachaud et al. [1] measured air pressure in an unsaturated stratified sand column by connecting a pressure transducer. The transducer was connected by a 'scanivalve' either to the external atmosphere or to the soil pore gas phase by using a hypodermic needle inserted through the column wall. During the infiltration of water, excess gas pressure built up at the advancement of the wetting front can be determined by the pressure transducer. Although the size of hypodermic needle is small, it is still permeable to both water and gas since there is no stopper at the end of the needle to separate water and gas. This means that water can still flow from the soil to the gas pressure measuring system during the

\footnotetext{
* Corresponding author: geobrucechen@163.com
} 
measuring process, causing the change of soil water content surrounding the end of the needle.

To improve the reliability for measuring air pressure in wet soils, Sakaki et al. [7] has tested seven different porous materials for air pressure measurement in soils that were initially water saturated. Among these seven porous materials, only nonwoven PTFE sheet was not required to be treated because PTFE is hydrophobic by itself. However, the performance of PTFE in measuring the air pressure during drainage was not tested in their study. Furthermore, this type of PTFE membrane is only subjected to low gas pressure (i.e., less than $10 \mathrm{kPa}$ ) which cannot be used to measure the gas pressure up to $50 \mathrm{kPa}$ which is the extreme landfill gas pressure under the landfill cover [6]. In this study, a novel pore gas pressure transducer was developed to measure the gas pressure in unsaturated soil within a wide range of water content. The performance of the gas pressure transducer was evaluated by a series of permeation tests.

\section{Material and methods}

In this section, the components of the developed pore gas pressure transducer and the measuring mechanism are firstly presented. The transducer was calibrated with a known pressure applied by a pressure apparatus. Subsequently, the performance of the transducer was evaluated through an element test where a soil at relative high water content was utilized.

\subsection{Components of the pore gas pressure transducer}

The newly developed pore gas pressure transducer mainly contains two parts: electrical gas pressure sensor package and an integrated membrane filter. These two parts are connected via a double layer tube. Inner tube is made of a hollow stainless steel with an inner diameter of $1 \mathrm{~mm}$ and outer diameter of $1.8 \mathrm{~mm}$. Outer tube is made from a flexible plastic. The function of the inner tube is to convey the gas pressure from the soil to the electrical gas pressure sensor. To minimize the effect of deformation of tube on gas pressure measurement, stainless steel is selected to form the tube. The outer tube has two main functions. One is to facilitate the connection between gas pressure sensor package and integrated filter. The other is to reduce the effect of the fluctuation of outer temperature on gas pressure measurement by forming an air layer between inner tube and outer tube. The measuring range of the electrical gas pressure sensor package is from $-100 \mathrm{kPa}$ to $100 \mathrm{kPa}$ (gauge pressure) and the accuracy is $0.2 \% \mathrm{FS}$.

The integrated filter package includes a plexiglass cylinder base, a flexible plastic buffer, a PTFE hydrophobic filter and a fixed ring as shown in Figure 1. The outer diameter of the plexiglass cylinder is $14 \mathrm{~mm}$. The thickness of the cylinder is $3 \mathrm{~mm}$. This material has

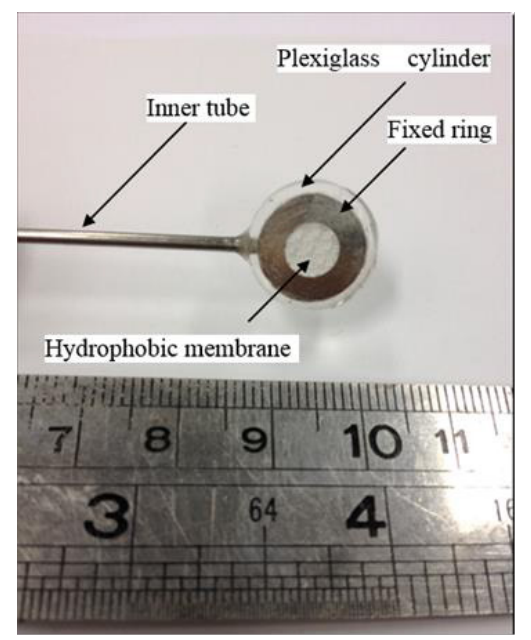

Fig. 1. Overview of the pore gas pressure transducer.

high hardness and good capability to prevent the effect of soil stress on the gas measuring system. The stopper was pasted on the outlet of a stainless tube which was connected the gas cell inside the plexiglass cylinder base. The PTFE hydrophobic filter is able to prevent water entry when the water pressure is lower than the bubble point pressure of the filter. The bubble point is the minimum pressure required to force water out of the pores. Essentially, the bubble point pressure is determined by the pore diameter. Due to the special feature of hydrophobic filter, pore gas pressure in wet and high compaction soils can be effectively measured while water cannot enter the gas pressure measuring system.

\subsection{Quality evaluation test of the filter used in the gas pressure transducer}

The objective of the quality evaluation test is to assess the performance of the filter in preventing water entry under certain water pressures whereas gas can pass the filter. In this test, the PTFE hydrophobic membrane is clamped between two steel plates. Two O-rings were installed between the membrane and each plate. One drainage hole was drilled in the centre of each plate to connect with tubes. One tube with one end connecting a water column (200 $\mathrm{mm}$ in height) to the membrane and the other end is connected with gas pressure source produced by a compressed air machine. Another tube is open to the atmosphere (i.e., $P_{g}=0$ ). The gas pressure of $50 \mathrm{kPa}$ was applied to the water column for 10 days. During the test, no water movement was observed in the tube but gas flow was captured from the downstream, indicating a good quality of water preventing of the filter.

\subsection{Calibration of the pore gas pressure transducer}

After the assembly of the gas pressure transducer and the quality assessment test, the developed gas pressure transducer together with an air pressure sensor were put into a plexiglass chamber (see Figure 2). Gas pressure ranged from 0 to $50 \mathrm{kPa}$ was gradually applied to the 
chamber step by step. Each step with an increase in gas pressure of $10 \mathrm{kPa}$ was utilized. When the pressure reaches equilibrium, the step is considered to be the end. Then gas pressure was gradually released from $50 \mathrm{kPa}$ to 0 step by step.



Fig. 2. Setup of the calibration system.

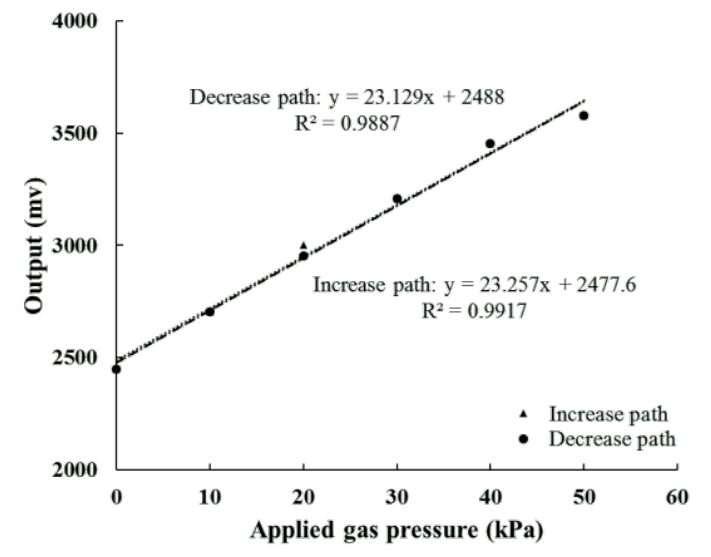

Fig. 3. Calibration of the pore gas pressure transducer.

Figure 3 shows the calibration result of the developed pore gas pressure transducer. The curve contains two paths: load path and unload path. The linearity of these two curves clearly demonstrated that the excellent degree of accuracy of the developed pore gas pressure transducer as compared to a pressure gauge. The consistency of the load and unload path during applying gas pressure shows that the developed gas pressure transducer has a small magnitude of hysteresis. In addition, the applied gas pressure ranged from 0 to $50 \mathrm{kPa}$ explains that the gas pressure under the range from 0 to $50 \mathrm{kPa}$ has no effect on the performance of the PTFE membrane. This means the developed pore gas pressure transducer is able to be used in the pressure measurement within a higher range (i.e., 0$50 \mathrm{kPa})$.

\section{Performance evaluation procedure of the pore gas pressure transducer}

After calibration of the developed gas pressure transducer, its performance in measuring gas pressure in wet soil was verified in this section. Completely decomposed granite (CDG) was compacted in a soil column with an inner diameter of $80 \mathrm{~mm}$ and height of $50 \mathrm{~mm}$. The maximum dry density and optimum water content by weight of CDG are $1870 \mathrm{~kg} / \mathrm{m}^{3}$ and $13 \%$, respectively. Based on the measured particle-size distribution and Atterberg limits, CDG is classified as silty sand (SM) according to the Unified Soil Classification System (USCS). CDG was compacted at relative compaction (RC) of $70 \%$. The index properties of CDG are summarized in Table 1. The compaction gravimetric water content (GWC) is around $22 \%$. CDG with a thickness of $40 \mathrm{~mm}$ was compacted at 5 lifts. Porous stone was placed on each end of the column to distribute gas pressure. Downstream pressure gauge was used to monitor the applied air pressure. Upstream pressure gauge was used to measure gas pressure in the lower space after gas passing through the soil specimen. A developed gas pressure transducer was installed in the middle of the compacted clay. A gas pressure step of 10 $\mathrm{kPa}$ was applied to the column from 0 to $50 \mathrm{kPa}$. Gas pressure in soil was measured by the developed gas pressure transducer at a time interval of $10 \mathrm{~min}$.

Table 1. Basic properties of the soil.

\begin{tabular}{|c|c|}
\hline Property & Value \\
\hline Specific gravity & 2.60 \\
\hline Liquid limit (\%) & 24 \\
\hline Plastic limit (\%) & 18 \\
\hline Plasticity index (\%) & 1870 \\
\hline $\begin{array}{c}\text { Maximum dry density } \\
\left.\text { (kg/m }{ }^{3}\right)\end{array}$ & 13 \\
\hline $\begin{array}{c}\text { Optimum water content (\%) } \\
\text { Saturated water coefficient of } \\
\text { permeability (m/s) } \\
\text { (The water permeability } \\
\text { corresponds to soil density of } \\
\text { 1309 kg/m } \text { a }^{3} \text { at degree of } \\
\text { compaction of 70\%) }\end{array}$ & $2.2 \times 10^{-6}$ \\
\hline
\end{tabular}

Figure 4 shows the variation of upstream pressure, measured gas pressure by the developed pore gas pressure transducer and downstream pressure with time. It can be seen from the figures that gas penetrates into the soil gradually with time. The gas pressure was captured first by the developed pore gas pressure transducer installed in the middle height of soil specimen and then by the upstream pressure gauge after a certain time period. The increasing reading of upstream pressure gauge indicates that gas reaches the upstream end of the 




(a)

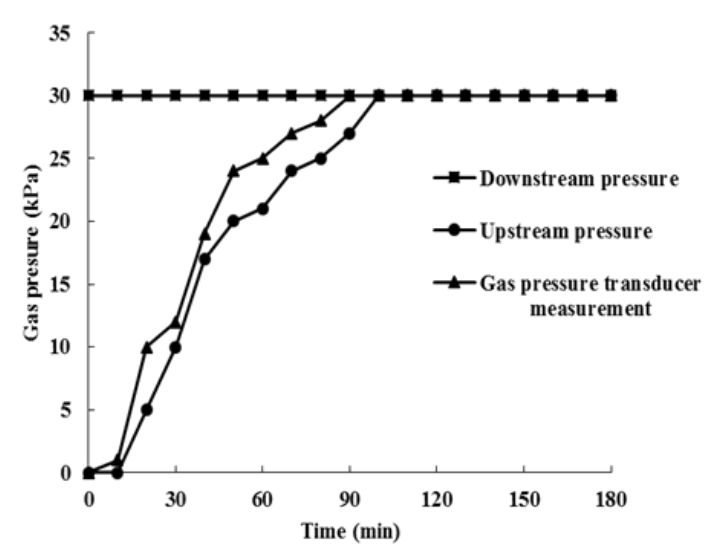

(b)

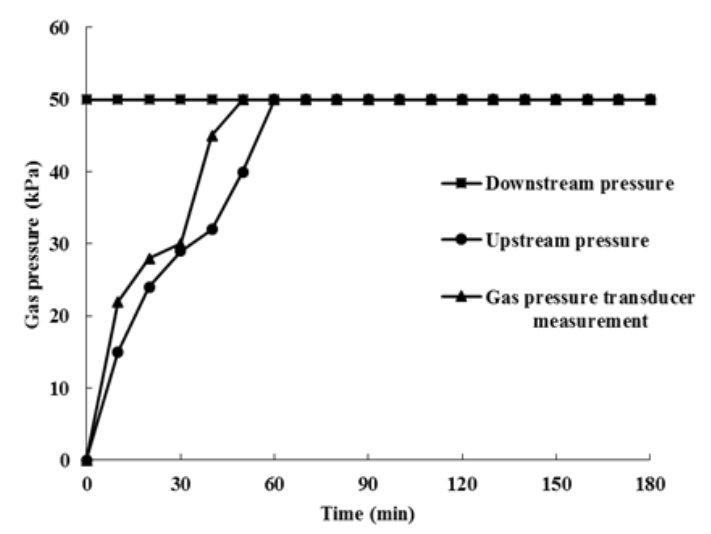

(c)

Fig. 4. Gas pressure variations with time (In $\mathrm{CDG}$ : $\mathrm{RC}=70 \%$, $\mathrm{GWC}=22 \%$ ): (a) apply gas pressure of $10 \mathrm{kPa}$; (b) apply gas pressure of $30 \mathrm{kPa}$; (c) apply gas pressure of $50 \mathrm{kPa}$.

soil column driven by the gas pressure gradient and accumulates with time.

The gas pressure was captured first by the developed pore gas pressure transducer installed in the middle height of soil specimen and then by the upstream pressure gauge after a certain time period. The increasing reading of upstream pressure gauge indicates that gas reaches the upstream end of the soil column driven by the gas pressure gradient and accumulates with time.

At the beginning of the applied gas pressure, the measured gas pressures of pore gas pressure transducer and upstream pressure gauge are zero. After a period of time, the soil gas pressure increases gradually and reaches the steady state at the end. The reached same pressure of upstream side as the downstream side represents the achievement of steady state. Based on the Darcy's law, the air pressure should be same eventually in the aircontinuous path in an unsaturated porous medium when there is no pressure gradient. The same pressure value measured by the pore gas pressure transducer as the upstream side and downstream side implies that the high degree of accuracy of the developed pore gas pressure transducer in measuring the gas pressure in wet soil. It can be seen from the figures, different applied pressures in the downstream side of the column generate a different time period for gas flow from downstream side to upstream side. This means the gas pressure needs time to displace soil water in order to penetrate a much deeper location under different gas pressure gradients. For instance, the measured gas pressure by the pore gas pressure transducer increased from 0 at the time of $10 \mathrm{~min}$ to $3 \mathrm{kPa}$ at the time of $20 \mathrm{~min}$. Furthermore, higher gas pressure gradient produces a shorter time period for gas penetrating to the location of the pore gas pressure transducer.

\section{Conclusions}

A pore gas pressure transducer was developed to measure air pressure in compacted soils with high water content. This pore gas pressure transducer was calibrated and verified using experiment. The results show that this pore gas pressure transducer has a relatively fast response to the air pressure change in compacted soils and can be used in the gas flow tests.

This research project is financially supported by National Natural Science Foundation of China (Grants No. 51578196 and 51808171), China Postdoctoral Science Foundation (Grant No. 2016M591536), Natural Science Foundation of Guangdong Province (Grant No. 2016A030310368) and Shenzhen Science and Technology Innovation Commission (No. JCYJ20170307150330877).

\section{References}

1. G. Vachaud, M. Vauclin, D. Khanji, M. Wakil, Water Resour., 9, 1, 160-173 (1973)

2. L. Prunty, J. Bell, Soil Sci. Soc. Am. J, 71, 5, 14731475 (2007)

3. F. J. Maciel, J. F. T. Jucá, Waste Manage, 31, 5, 966977 (2011)

4. D. M. Sun, Y. G. Zang, S. Semprich, Transport Porous Med, 107, 3, 821-841 (2015)

5. D.G. Fredlund, H. Rahardjo, Soil mechanics for unsaturated soils (John Wiley \& Sons, 1993)

6. H. Y. Wei, Ph.D. dissertation (Zhejiang University, Hangzhou, 2007)

7. T. Sakaki, A. Limsuwat, T. H. Illangasekare, Vadose Zone J., 10, 2, 706-715 (2011) 